\title{
New theorems in approximation theory
}

\author{
Nabaa Mery Kasim*
}

Date of acceptance 12/5/2009

\begin{abstract}
:
The aim of this paper is to prove some results for equivalence of moduli of smoothnes in approximation theory, we used a"non uniform" modulus of smoothness $\varpi_{\phi}^{k}(f, t)=\sup _{0<h \leq t}\left\|\Delta_{\beta}^{k}(f, x)\right\|_{p}$ and the weighted Ditzian -Totik moduli of smoothness $\varpi_{k, v}^{\psi}(f, t)=\sup _{0<h \leq t}\left\|\psi(.)^{v} \Delta_{h \psi(.)}^{k}(f, x)\right\|_{p} \quad$ in $\quad L_{p}[-1,1], 0<p \leq \infty$ by spline functions ,several results are obtained.For example, it shown that, for any $1 \leq \phi \leq r+1,1 \leq p \leq \infty \quad$ the inequality $\quad|J|^{v} \varpi_{\phi-v}^{k}\left(S^{(v)}, J\right)_{p} \approx \varpi_{\phi}^{k}(S, J)_{p}$, $1 \leq v \leq \min [\phi, m+1]$ is satisfied ,finally, similar result for chebyshev partition and weighted Ditzian -Totik moduli of smoothness are also obtained.
\end{abstract}

Key words: Univariate splines, moduli of smoothness, "non uniform" modulus of smoothness, weighted Ditzian -Totik moduli of smoothness.

\section{Introduction:}

Equivalence moduli of smoothness was proved by $\mathrm{Hu}$ and $\mathrm{Yu}$ [1] and by $\mathrm{Hu}$ [2] for uniform and quasi-uniform partition, respectively. In fact, if we set $m=k-1$, then corollary (1.5) (with an additional restriction $k \leq r$ ) becomes theorem 1 in [2] ,also in the case $k=r+1$ and $m=r-1$, corollary (1.5) follow from theorem 2 of [2] . [3]

A function $S$ is called a spline if :

1- the domain is an interval [a,b].

2- $S, S^{\prime}, S^{\prime \prime}, \ldots, S^{(r-1)}$ are all continuous function on $[\mathrm{a}, \mathrm{b}]$

3- there are point (the knot of $S$ )such that $a<Z_{0}<Z_{1}<\ldots<Z_{n-1}<Z_{n}=b$ and such that $S$ is a polynomial of degree $\leq r$ on each subintrrval $\left[Z_{i}, Z_{i+1}\right]$

Usually piecewise polynomials from $S_{r}\left(Z_{n}\right)$ are called "splines" if they possess continuous $(r-1)$ st derivatives .We emphasize that if $S \in S_{r}\left(Z_{n}\right)$ and $m \in N$ then $S^{(m)} \in \delta_{\max \{r-m, o\}}\left(Z_{n}\right)$ and $S^{(m)}=0$ a.e if $m \geq r+1$.

Now a moduli of smoothness are intended for mathematicians working in approximation theory ,numerical analysis and real analysis .Measuring the smoothness of function by differentiability is too crude for many purposes in approximation theory . more suitable measurement are

*Math .Department/College of Education /Abn-A provided by maitham/ University of Baghdad 
$L_{p}(J), 0<p \leq \infty$, denotes the space of all measurable function $f$ on $J$ Such that $\|f\|_{L(J)}<\infty$.

The kth symmetric of $f$ is given by

$\Delta_{\beta}^{-k}(f, x,[a, b])= \begin{cases}\sum_{i=0}^{k}(-1)^{k-i}\left(\begin{array}{l}k \\ i\end{array}\right) f(x+i \beta) & \text { if }[x, x+k \beta] \subset[a, b] \\ 0 & \text { other wise }\end{cases}$

then the kth "non uniform" modulus of smoothness of $f \in L_{p}[-1,1], 0<p \leq \infty$ is

by

$$
\varpi_{\phi}^{k}(f, t)=\sup _{0<h \leq t}\left\|\bar{\Delta}_{\beta}^{k}(f, x)\right\|_{p}
$$

Where

$\beta=\beta(x, h)=\sqrt{1-x^{2}} h+h^{2}, \phi(x)=\sqrt{1-x^{2}}$ and

The weighted Ditzian -Totik moduli of smoothness of a function $f \in L_{p}[-1,1], 0<p \leq \infty$ is define by $\varpi_{k, v}^{\psi}(f, t)=\sup _{0<h \leq t}\left\|\psi(.)^{v} \Delta_{h \psi(.)}^{k}(f, x)\right\|_{p},[5]$

Where $\psi(x)=\sqrt{1-x^{2}}$

A partition $Z_{n}=\left\{Z_{0}, \ldots Z_{n}\right\}=$ $-1<Z_{0}<Z_{1}<\ldots<Z_{n-1}<Z_{n}=1$ of the interval $[-1,1]$ denote the scale of parition $Z_{n}$ by $\theta=\theta\left(Z_{n}\right)=\max \frac{\left|J_{j} \pm 1\right|}{\left|J_{i}\right|}$ where $\quad J_{j}=\left\lfloor Z_{j}, Z_{j+1}\right\rfloor \quad$ with $Z_{j}=-1, j<0$ and $Z_{j}=1, j>n$.

\section{Auxiliary results}

The following properties of the moduli of smoothness are well known[6],[7]

(i) for

$f \in L_{p}(J), 0<p \leq \infty$, we have $\omega_{k+1}(f, t, J)_{p} \leq 2^{\max \{1,1 / p\}} \omega_{k}(f, t, J)_{p}, k \in N$

(ii)

$\omega_{k}(f, \lambda t, J)_{p} \leq C(k, p)(\lambda+1)^{k-1+\max \{1,1 / p\}}$

$\omega_{k}(f, t, J)_{p}, \lambda>0$

(iii) suppose that $f \in L_{p}[-1,1], 0<p<\infty$ and $k, \mu \in N$ then

$\sum_{j=0}^{n-\mu-1} \omega_{k}\left(f, \bigcup_{i=j}^{j+\mu} J_{i}\right)_{p}^{p} \leq \begin{cases}c(k, \Delta, \mu, p) \omega_{k}\left(f, n^{-1}\right)_{p}^{p} & \text { if } \mathrm{z}_{\mathrm{n}}=u_{n}^{\Delta} \\ c(k, \mu, p) \omega_{k}^{\varphi}\left(f, n^{-1}\right)_{p}^{p} & \text { if } \mathrm{z}_{\mathrm{n}}=t_{n}\end{cases}$

Lemma .1 (Whitneys inequality

). [8]

For any $f \in L_{p}[a, b], 0<p \leq \infty$ such that $q_{k-1} \in \Pi_{k-1}$ such that

$\left\|f-q_{k-1}\right\|_{L_{p}[a, b]} \leq C \omega_{k}(f,[a, b])$.

\section{Lemma .2}

For any polynomial $q_{k} \in \Pi_{k}$ , $0<p \leq \infty$ and intervals $I$ and $J$ such that $I \subseteq J$ we have

$$
\begin{aligned}
& |J| \frac{1}{p}\left\|q_{r}^{(v)}\right\|_{L_{\infty}(J)} \approx\left\|q_{r}^{(v)}\right\|_{L_{p}(J)} \leq \\
& C(r,|J| /|I|, p)|J|^{-v}\left\|q_{r}\right\|_{L_{p}(I)}, 0 \leq v \leq r
\end{aligned}
$$

\section{The main result}

The following theorem is not satisfied for all $f \in L_{p}, 0<p<\infty$ but satisfied for spline functions

\section{Theorem .1 (local estimates)}

Let $S \in S_{r}\left(Z_{n}\right), r \in N \quad$ and $J=\left[Z_{M_{1}}, Z_{M_{2}}\right]$ with $M_{2}-M_{1} \leq c_{0}$ for some constant $c_{0}$

Then for $1 \leq \phi \leq r+1,0 \leq p \leq \infty$ we have 
$\varpi_{\phi-v}^{k}\left(S^{(v)}, J\right)_{p} \leq c\left(r, \theta, c_{0}, p\right)|J|^{-v} \varpi_{\phi}^{k}(S, J)_{p}$ for all $v=1,2, \ldots, \phi$.

\section{proof:}

Every where in this proof $q_{k-1}$ denote a polynomial

of $\leq k-1$ э $\left\|s-q_{k-1}\right\|_{L_{p(J)} \leq C \varpi_{\phi}^{k}(s, J)}{ }_{p}$

for any $1 \leq v \leq \phi$. denoting $s_{j}=s / J_{i}$ and using [(2.5),[7 ] ] we have

$$
\begin{aligned}
& \left|\Delta_{h}^{k-v}\left(s^{(v)}, x, J\right)\right| \leq\left|\overleftarrow{\Delta}_{p}^{k-v}\left(s^{(v)}, x, J\right)\right| \text { since } \\
& \varpi_{\phi}^{k}(f, t) \approx \omega_{\phi}^{k}(f, t)_{\infty} \\
& =\left|\overleftarrow{\Delta}_{p}^{k-v}\left(s^{(v)}-q_{k-1}^{(v)}, x, J\right)\right| \\
& \leq 2^{k-v}\left\|S^{(v)}-q_{k-1}^{(v)}\right\|_{\infty}(J) \\
& \leq C(k) \max _{M_{1} \leq j \leq M_{2}-1}\left\|S^{(v)}-q_{k-1}^{(v)}\right\|_{L_{\infty}}\left(J_{j}\right) \\
& \leq C(r, p) \max \left|J_{j}\right|^{-v-\frac{1}{p}}\left\|s_{j}-q_{k-1}\right\|_{L_{p}}(J j) \\
& \leq C\left(r, \theta, c_{0}, p\right)\left|J_{j}\right|^{-v-\frac{1}{p}}\left\|s-q_{k-1}\right\|_{L_{p}\left(J_{j}\right)} \\
& \leq C\left(r, \theta, c_{0}, p\right)\left|J_{j}\right|^{-v-\frac{1}{p}} \varpi_{\phi}^{k}(s, J)_{p} .
\end{aligned}
$$

Corollary .2 (equivalaence of moduli of smoothness)

Let $S \in S_{r}\left(Z_{n}\right) \bigcap C^{m}[-1,1], r \in N$ and $J=\left\lfloor Z_{M_{1}}, Z_{M_{2}}\right\rfloor$ with $M_{2}-M_{1} \leq c_{0}$ for some constant $c_{0}$

Then for $1 \leq \phi \leq r+1,1 \leq p \leq \infty$ we have $|J|^{v} \varpi_{\phi-v}^{k}\left(S^{(v)}, J\right)_{p} \approx \varpi_{\phi}^{k}(S, J)_{p}$, $1 \leq v \leq \min [\phi, m+1]$
Equivalaence constants above depend only on $r, \theta, c_{0}$.

Suppose that $\delta_{\max }=\delta_{\max }\left(Z_{n}\right)=\max _{0 \leq j \leq n-1}\left|J_{j}\right| \quad$ and $\delta_{\text {min }}\left(Z_{n}\right)=\min _{0 \leq j \leq n-1}\left|J_{j}\right|$ we say that $Z_{n} \quad$ is $\quad \Delta$-quasi uniform if $\Delta=\delta_{\max } / \delta_{\min }$ is bounded by a constant independen of $n$, and denote such partition by $u_{n}^{\Delta}$, if $Z_{n}=u_{n}^{\Delta}$ then $2 /(n \Delta) \leq \delta_{\min } \leq 2 / n<\delta_{\max }<2 \Delta / n$ and $\theta\left(Z_{n}\right) \leq \Delta$

Therefore $\quad \delta_{\text {min }} \approx \delta_{\text {max }} \approx n^{=1} \quad$ with Equivalaence constants depending only on $\Delta$.

\section{Theorem.3}

\section{partition)}

Let $u_{n}^{\Delta}, n \in N$ be a quasi uniform partition of $[-1,1]$ and let $S \in$ $S_{r}\left(u_{n}^{\Delta}\right), r \in N \quad$ then for any $1 \leq \phi \leq r+1,0<p \leq \infty$ we have $\varpi_{\phi-v}^{k}\left(S^{(v)}, n^{-1}\right)_{p} \leq c(r, \Delta, p) n^{v} \varpi_{\phi}^{k}\left(S, n^{-1}\right)_{p}$ for all $v=1,2, \ldots, \phi$.

\section{proof}

Since $u_{n}^{\Delta}$ is a $\Delta$ - quasi uniform $\delta_{\text {max }} \approx \delta_{\text {min }} \approx\left|J_{i}\right| \approx n^{-1} \quad$ for all $0 \leq j \leq n-1 \quad$ with equivalence constants depending only $\Delta$. Using (2.2) we have

$$
\begin{aligned}
& \varpi_{\phi-v}^{k}\left(s^{(v)}, n^{-1}\right)_{p}=\varpi_{\phi-v}^{k}\left(s^{(v)}, \frac{\phi}{n \delta_{\min }}, \frac{\delta_{\min }}{\phi}\right) \\
& \leq C(\phi, \Delta, p) \varpi_{\phi-v}^{k}\left(s^{(v)}, \delta_{\min } / \phi\right)_{p}
\end{aligned}
$$

Now, for any $0<\beta<\delta_{\min } / \phi$ and $x \in J_{j}, 0 \leq j \leq n-1 \quad$ such that $\Delta_{\beta}^{\phi-v}\left(s^{(v)}, x\right) \neq 0$ 
All $x-(\phi-v) \beta / 2+i \beta, 0 \leq \mathrm{i} \leq \phi-\mathrm{v} \quad$ are either in $J_{j-1} \bigcup J_{j}$ or $J_{j} \bigcup J_{j+1}$

$$
\begin{gathered}
\varpi_{\phi-v}^{k}\left(s^{(v)}, \delta_{\min } / \phi\right)_{p}^{p}=\sup _{0 \leq \beta \leq \delta_{\min } / \phi}\left\|\bar{\Delta}_{\beta}^{k(\phi-v)}\left(s^{(v)}, .\right)\right\|_{p}^{p} \\
=\sup _{0 \leq \beta \leq \delta_{\min }} \sum_{\phi}^{n-1}\left\|\bar{\Delta}_{\beta}^{k(\phi-v)}\left(s^{(v)}, .\right)\right\|_{p}^{p}
\end{gathered}
$$$$
\leq 2
$$

$\sup _{0 \leq \beta \leq \delta_{\min } / \phi} \sum_{j=0}^{n-1}\left\|\Delta_{\beta}^{k(\phi-v)}\left(s^{(v)}, ., J_{j-1} \cup J_{j}\right)\right\|_{L_{p}\left(J_{j-1} \cup J_{j}\right)}^{p}$

$\leq 2 \sum_{j=1}^{n-1} \varpi_{\phi-v}^{k}\left(s^{(v)}, J_{j-1} \bigcup J_{j}\right)_{p}^{p}$

$\leq C(r, \Delta, p) \sum_{j=1}^{n-1}\left|J_{j-1} \bigcup J_{j}\right|^{-v p} \varpi_{\phi}^{k}\left(s, J_{j-1} \bigcup J_{j}\right)_{p}^{p}$

$\leq C(r, \Delta, p) n^{p v} \sum_{j=1}^{n-1} \varpi_{\phi}^{k}\left(s, J_{j-1} \bigcup J_{j}\right)_{p}^{p}$

$\leq C(r, \Delta, p) n^{p v} \varpi_{\phi}^{k}\left(s, n^{-1}\right)_{p}^{p}$.

Where the last inequality follow from (2.3)

\section{Corollary .4}

Let $u_{n}^{\Delta}, n \in N$ be a quasi uniform partition of $[-1,1]$ and let $S \in$ $S_{r}\left(u_{n}^{\Delta}\right) \bigcap C^{m}[-1,1], r \in N, 0 \leq m \leq r-1$ then for any $1 \leq \phi \leq r+1,1 \leq p \leq \infty$ we have $n^{-v} \varpi_{\phi-v}^{k}\left(S^{(v)}, n^{-1}\right)_{p} \approx \varpi_{\phi}^{k}\left(S, n^{-1}\right)_{p}$, $1 \leq v \leq \min [\phi, m+1]$

Equivalaence constants above depend only on $r, \Delta$. .
Let $Z_{n}$ is a chebyshev partition , if $Z_{n}=t_{n}=\left(t_{i}\right)_{i=0}^{n} \quad$ where $t_{i}=\cos \left(\frac{(n-i) \Pi}{n}\right), 0 \leq i \leq n$.

\section{Theorem.5 (chebyshev knote)}

Let $t_{n}$ be a chebyshev partition of $[-1,1]$ if $s \in S_{r}\left(t_{n}\right), r \in N$ then, for any $1 \leq \psi \leq r+1,0<p<1$ we have $\varpi_{\psi-v}^{k}\left(S^{(v)}, n^{-1}\right)_{p} \leq c(r, p) n^{v} \varpi_{\psi}^{k}\left(S, n^{-1}\right)_{p}$ for all $1 \leq v \leq \psi$.

\section{Proof:}

Chebyshev knote: let $J_{j, \phi-v}=\left[t_{j-3(\psi-v)}, t_{j+4+3(\psi-v)}\right], \quad$ denote $J_{j, \psi-v}$, and , for each $0 \leq j \leq n-1$

Let $q_{j} \in \Pi_{\phi-v-1}$ be such that $\left\|s^{(v)}-q_{j}\right\|_{L_{p}\left(J_{j}\right)} \leq C \varpi_{\phi-v}\left(s^{(v)}, J_{j}\right)_{p}$

[6 ], and we assume that $q_{j}=0$ if $v=\psi$. Then using theorem 3.1 and the inequality $\quad \psi(x) \leq n\left|J_{j}\right|, x \in J_{j}$, we have

$$
\begin{aligned}
& \omega_{k-v, v}^{\psi}\left(s^{(v)}, n^{-1}\right)_{p}=\sup _{0<h<n^{-1}} \| \psi^{v} \Delta_{h \psi}^{\left.k-v_{(s}{ }^{(v)}, .\right) \|_{p}} \\
& = \\
& \sup _{0<h<n^{-1}} \sum_{j=0}^{n-1}\left\|\psi^{v} \Delta_{h \psi}^{k-v}\left(s^{(v)}, .\right)\right\|_{L_{p}\left(J_{j}\right)} \\
& \leq \sum n^{v p}\left|J_{j}\right|^{v p} \\
& \sup _{0<h<n^{-1}}\left\|\psi^{v} \Delta_{h \psi}^{\left.k-v_{\left(s^{(v)}\right.}-q_{j}, .\right)}\right\|_{L_{p}\left(J_{j}\right)} \\
& \leq C(k, p) n v \sum_{j=0}^{v p}\left|J_{j}\right|^{v p}\left\|s^{(v)}-q\right\|_{j} \|_{p}\left(J_{j}\right) \\
& \leq C(k, p) n^{v p} \sum_{j=0}^{n-1}\left|J_{j}\right|^{v p} \omega_{k-v, v}\left(s^{(v)}, J_{j}\right)_{p} \\
& \leq C(r, p) n^{v p} \sum_{j=0}^{n-1} \omega_{k}\left(s, J_{j}\right)_{p} \\
& \leq C(r, p) n^{v p} \omega_{k}^{\psi}\left(s, n^{-1}\right)_{p}
\end{aligned}
$$


where the last inequality follows from ( 2.3)

\section{Corollary.6}

Let $\quad s \in S_{r}\left(t_{n}\right) \bigcap C^{m}[-1,1]$ $r \in N, 0 \leq m \leq r-1$ then, for any $1 \leq \psi \leq r+1,0<p<1 \quad$ we have $n^{-v} \varpi_{\psi-v}^{k}\left(S^{(v)}, n^{-1}\right)_{p} \approx \varpi_{\psi}^{k}\left(S, n^{-1}\right)_{p}$ for all $1 \leq v \leq \min \{\psi, m+1\}$.

Equivalaence constants above depend only on $r$.

\section{References:}

[1] Hu.Y and Yu.X.M.1995.Discrete modulus of smoothness of splines with equally spaced knots .SIAMJ.Numer .Anal.(32):14281435.

[2] Hu.Y.1999.Some theorems of the moduli of smoothness in approximation theory.J.Aprox.282293.
[3] Beatsone. R.K. 1981.Convex approximation by spline. SIAMJ. Math.(12):549-559.

[4] DeVore.R.A. and Totik.V.1987. Moduli of smoothness.New york .Sprinnger-Verlag.(3):201-215.

[5] Kopotun.K,Leviatan .D and Shevchuk .2006.Coconvex approximation in the uniform norm,Acta.mat.Hungar.110(12):117-151.

[6]DeVore.R.A and Lorentz.G.G.2003. Constructive approximation . Berlin .springer-verlag,vol.(4): 303-307.

[7] Petrushev.P.P and Popov.V.A. 1987.Rational approximation of real functions. Cambridge University press.vol.28(5):12311236.

[8] Kopotun.K.A. 2001. Whitney theorem of interpolatory type for $\mathrm{K}$-monotone .constructive $.17: 307-317$.

\section{مبرهنات جديدة في نظرية التقريب

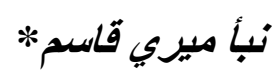

*قنم الرياضيات /كلية التربية /ابن الهيثم/جامعة بغداد

الهدف من هذا البحث هو برهـان نتائج للتكافؤات بين مقايس النعومة في نظريـة التقريب استخدمنا مقياس

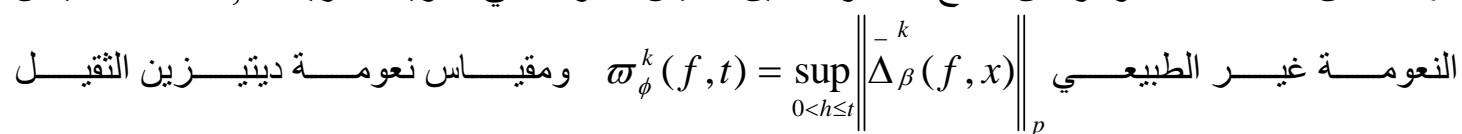

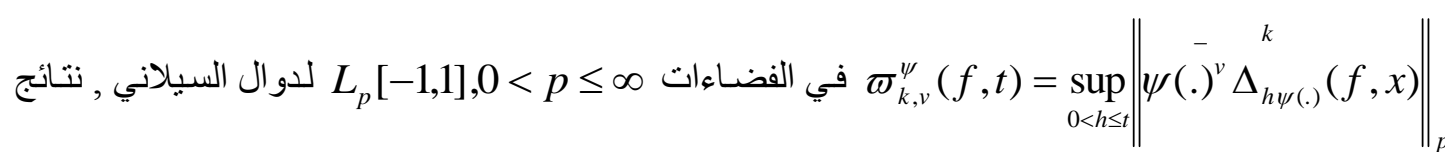

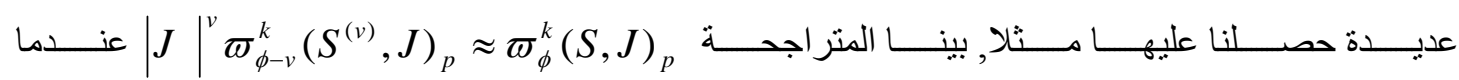
1 $1 \leq v \leq \min [\phi, m+1]$ متحققة واخيرا بينـا تجزئة شييشيف متحققة ايضا باستخدام مقياس نعومة ديتزين الثقيل. 